\title{
Direct Numerical Simulations of Membrane Wings at Low Reynolds Number
}

\author{
Sonia Serrano-Galiano* and Richard D. Sandberg ${ }^{\dagger}$ \\ Faculty of Engineering and the Environment, University of Southampton, Southampton SO17 1BJ, U.K.
}

\begin{abstract}
Direct numerical simulations (DNS) of membrane wings at low Reynolds numbers were performed at different angles of attack using a new high-fidelity fluid-structure interaction computational framework. A newly implemented boundary data immersion method coupled to a membrane structural solver was used in a well validated DNS code. DNS performed at lower Reynolds number $\mathrm{Re}=\mathbf{2 5 0 0}$ agree well with reference data, validating the new numerical set-up. For higher Reynolds number $R \mathbf{e}=\mathbf{1 0 , 0 0 0 ,}$ where the membrane becomes unsteady and the flow motion becomes more chaotic, it is found that spectra of membrane deflection and pressure coefficient are still closely correlated. The computational set-up is employed for preliminary studies of the stability characteristics of separation bubbles with vortex shedding for membrane aerofoils. The evolution of initial perturbations introduced on the flow field were investigated recording the temporal growth of the spanwise Fourier modes of the perturbations. Regions of amplification of perturbations were identified for the case at higher Reynolds number and angle of attack.
\end{abstract}

\section{Introduction}

All existing natural flyers have a common key element for their high manoeuvrability and exceptional aerodynamics: their flexible wings. Particularly, for mammal flyers (i.e. bats) they consist of a membrane surface covered with different tissues that can continuously adjust the shape depending on the incoming air flow, providing controllability and stability. Therefore membrane wings become a very desirable solution for application to Micro-Air Vehicles (MAVs) design, especially due to the similarities in size and flight regime that these devices have with bats.

It is expected that these kind of vehicles can take advantage of the improvements that membrane wings have over traditional rigid wings such as weight reduction, improvement of lift characteristics, stall and diminution of the effect of atmospheric turbulence and gust on aerodynamic loads and flight stability, and may allow for beneficial aeroelastic tailoring and active control. ${ }^{1}$ The study and simulation of membrane wings provides a foundation to develop MAVs fitted with these types of wings. This study requires a combination of at least two disciplines: fluid dynamics and structural mechanics, applied to elastics bodies. Unlike for the rigid wing case, for membrane wings the behaviour of the flow around the body is directly affected by the change of the surface shape over time, and therefore the aerodynamic properties and performance of the wing are directly dependant on those changes. Vice versa, the structural deformations depend on the fluid dynamics. The influence of different aerodynamic and structural parameters such as angle of attack, pretension of the membrane or modulus of elasticity at low Reynolds numbers has been studied by performing computational simulations and analysis for a two-dimensional membrane wing immersed in a compressible viscous flow. ${ }^{2}$ Simulations extended to a higher transitional/turbulent Reynolds number using an implicit large eddy simulation method were presented by Gordnier and Attar. ${ }^{3}$ Other aspects of membrane wings such as the vortex dynamics and unsteady aerodynamic loads for plunge and combined pitch-plunge motions of the wing mounts has been addressed by Jaworski and Gordnier. ${ }^{1}$ Also, thrust generation and flow-field characteristics of a two-dimensional flapping flexible membrane with variable bending rigidities have been studied by Ferreira de Sousa and Allen. ${ }^{4}$ However, so far hydrodynamic stability or laminar separation bubbles studies have not been carried out in the context of membrane aerofoils at low Reynolds numbers

\footnotetext{
*PhD student, University of Southampton s.serrano-galiano@soton.ac.uk, AIAA student member.

$\dagger^{\dagger}$ Professor, University of Southampton, sandberg@soton.ac.uk, AIAA senior member.
} 
in a similar way to traditional rigid aerofoils. Since the membrane behaviour is intimately subjected to the flow dynamics close to the surface, the study of laminar separation and transition and the role of instability mechanisms on this flow configuration is essential for the understanding of fluid-membrane interaction. In particular, valuable conclusions from this study would be useful in terms of controllability of the wing shape to avoid possible unstable modes.

To that end, direct numerical simulations (DNS) of two dimensional membrane wings at low and moderate Reynolds number are carried out. A well validated DNS code was coupled to a non-linear structural solver together with a recently implemented boundary data immersion method. The solution of the 2D DNS cases are used as initial condition for three dimensional simulations and initial perturbations of the spanwise velocity are introduced in order to identify regions of instability.

\section{Governing Equations}

\section{A. Fluid solver}

The in-house DNS code solves the compressible Navier-Stokes equations. The flow is assumed to be an ideal gas with constant specific heat coefficients and the equations are nondimensionalized with the flow quantities in the free-stream and the membrane chord length. The non-dimensional continuity, momentum and the energy equations in tensor notation are:

$$
\begin{aligned}
\frac{\partial \rho}{\partial t} & +\frac{\partial}{\partial x_{k}}\left(\rho u_{k}\right)=0 \\
\frac{\partial}{\partial t}\left(\rho u_{i}\right) & +\frac{\partial}{\partial x_{k}}\left[\rho u_{i} u_{k}+p \delta_{i k}-\tau_{i k}\right]=0, \\
\frac{\partial}{\partial t}(\rho E) & +\frac{\partial}{\partial x_{k}}\left[\rho u_{k}\left(E+\frac{p}{\rho}\right)+q_{k}-u_{i} \tau_{i k}\right]=0,
\end{aligned}
$$

where the total energy is defined as $E=T /\left[\gamma(\gamma-1) M^{2}\right]+0.5 u_{i} u_{i}$. The stress tensor and the heat-flux vector are computed respectively as

$$
\tau_{i k}=\frac{\mu}{R e}\left(\frac{\partial u_{i}}{\partial x_{k}}+\frac{\partial u_{k}}{\partial x_{i}}-\frac{2}{3} \frac{\partial u_{j}}{\partial x_{j}} \delta_{i k}\right) \quad, \quad q_{k}=\frac{-\mu}{(\gamma-1) M^{2} \operatorname{PrRe}} \frac{\partial T}{\partial x_{k}} .
$$

The Prandtl number is assumed to be constant at $\operatorname{Pr}=0.72$, and $\gamma=1.4$. The molecular viscosity $\mu$ is computed using Sutherland's law, ${ }^{5}$ setting the ratio of the Sutherland constant over freestream temperature to 0.36867 . To close the system of equations, the pressure is obtained from the non-dimensional equation of state $p=(\rho T) /\left(\gamma M^{2}\right)$.

The compressible Navier-Stokes equations are solved using a high-order accurate numerical scheme applicable to general geometries. A five-point stencil fourth-order accurate standard central difference scheme with Carpenter boundary stencils ${ }^{6}$ is applied for the spatial discretization in the streamwise $(x)$ and the wall-normal $(y)$ directions and a pseudo-spectral approach is employed in the spanwise direction $(z)$. For the integration in time an ultra low-storage five-step fourth-order Runge-Kutta scheme ${ }^{7}$ is used. The stability of the code is enhanced by a skew-symmetric splitting of the non-linear terms. ${ }^{8}$ A sixth-order accurate high-wavenumber cut-off filter ${ }^{9}$ with a weighting of 0.2 is employed after every full Runge-Kutta cycle.

Non-physical numerical reflections from the boundaries are avoided by using characteristic boundary conditions. At the inflow they are applied in an integral formulation ${ }^{10}$ and for the outflow a zonal characteristic boundary condition ${ }^{11}$ is used to avoid spurious pressure-oscillations due to the passage of non-linear disturbances.

The discretized fluid equations are solved in an Eulerian Cartesian grid with resolution and size described in section III.

\section{B. Structural solver}

The structural model for the membrane used in this study is based on the model presented by Gordnier ${ }^{2}$ for a one-dimensional non-linear membrane under normal forces.

The governing equation in this case is as follows 


$$
\begin{gathered}
\rho_{s} h \frac{\partial^{2} w}{\partial t^{2}}+\rho_{s} C_{d} \frac{\partial w}{\partial t}-T_{s} \frac{\partial^{2} w}{\partial x^{2}}\left[1+\left(\frac{\partial w}{\partial x}\right)^{2}\right]^{-3 / 2}=\Delta p, \quad \text { where } \\
T_{s}=E_{s} h\left(\delta_{0}+\delta\right) \quad, \quad \delta=L-1 \quad, \quad L=\int_{0}^{1} \sqrt{1+\left(\frac{\partial w}{\partial x}\right)^{2}} d x
\end{gathered}
$$

In these equations $w$ is the displacement of the membrane and the parameters $\rho_{s}, h, C_{d}, T_{s}, E_{s}$ and $\delta$ are respectively the membrane density, thickness, damping coefficient, tension, modulus of elasticity and the length increase of the pre-strained membrane. It is assumed that the material properties are uniform along the membrane. Here all quantities are also nondimensionalized using the freestream density and velocity of the fluid and the chord length of the membrane. The pressure force term $\Delta p$ is defined as the difference of flow pressure between the lower and the upper surface of the membrane immersed in the flow. Therefore this term is responsible for coupling the flow behaviour and the membrane response.

In the case under study the membrane is assumed to be pinned at the two ends, with the boundary conditions being $w(x=0, t)=0$ and $w(x=1, t)=0$. A representation of this configuration is shown in Fig.1.

In the newly implemented structural solver the governing equation (5) involving a second derivative in time is rewritten as a system of two equations with just first derivatives in time. The first and second spatial derivatives are evaluated by means of the same spatial discretization scheme as the flow solver. The resulting system of equations discretized in space is advanced in time also using the same fourth-order Runge-Kutta method as used for the flow. After each time step an implicit penta-diagonal filter ${ }^{12}$ is used for stabilization. The discretized membrane equation is solved on a one-dimensional Lagrangian grid that follows the membrane deformation. Thanks to the used immersed boundary technique this grid is totally independent from the fixed flow grid, and both sets of computational points do not need to coincide at any time.

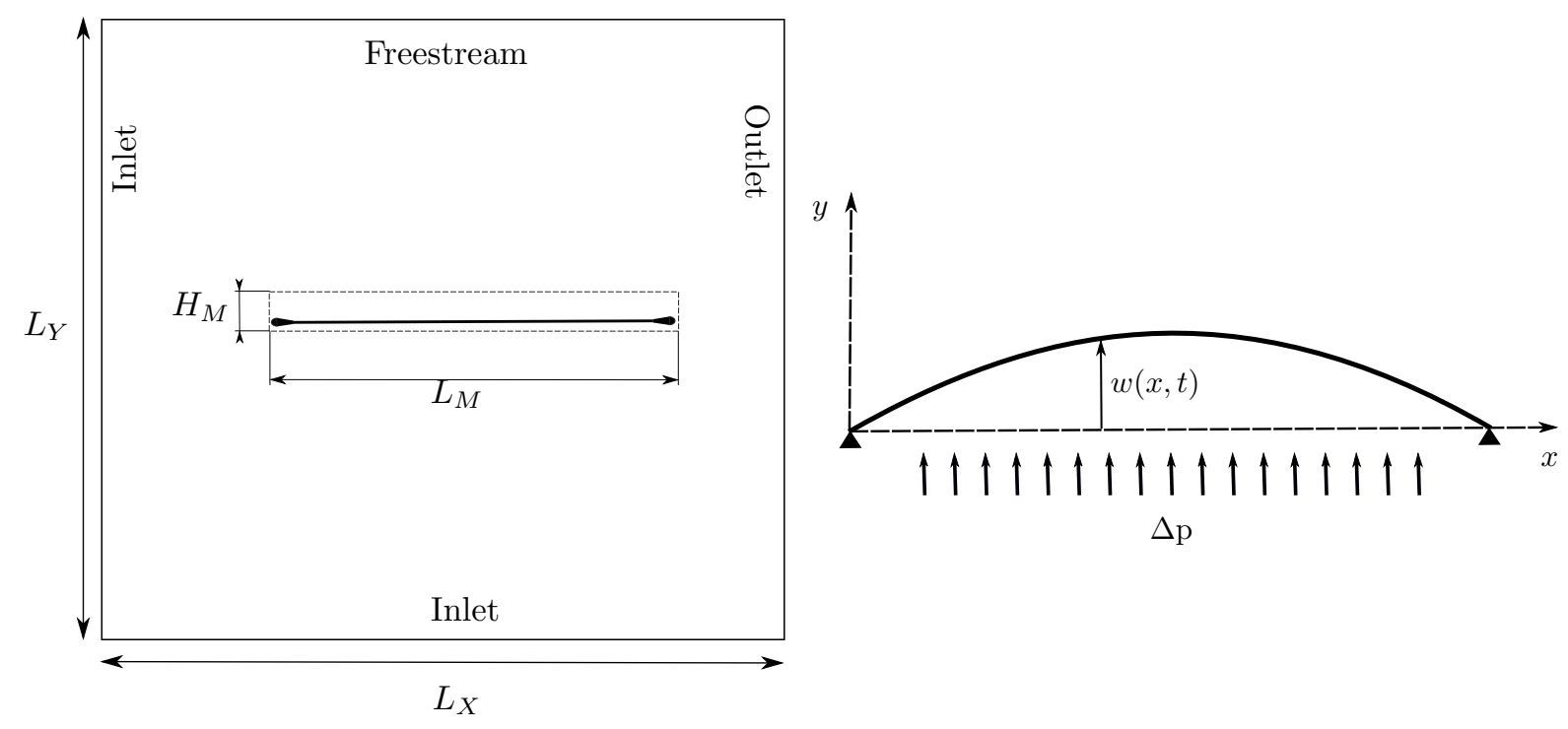

Figure 1: Left: Sketch of the computational domain. The dashed line rectangle delimits the Eulerian grid fine region. Right: Sketch of the membrane configuration, pinned at both ends.

\section{Fluid-Structure interaction coupling}

The fluid and the structural solver are coupled through the forcing pressure term $\Delta p$ in Eq.(5) and the deflection $w$ that it causes in the membrane. This coupling is achieved when at the beginning of each time step the pressure on the upper and lower surface of the membrane are transferred from the fluid to the structural solver via bilinear interpolation between grids. Then the deflection and velocity of the structure are calculated for the new time step. The new geometry of the immersed body is updated and transferred back to the fluid solver. The time step for DNS is very small in order to resolve the relevant time scales of the 
fluid solution, therefore it is assumed that the time lagging between pressure term and structural deflection is negligible.

The geometry and boundary conditions on the solid body immersed in the flow are imposed by means of a boundary data immersion method (BDIM) proposed by Weymouth and Yue ${ }^{13}$ which was recently extended and validated for the use in compressible flows by Schlanderer and Sandberg, ${ }^{14}$ implemented in the fluid-structure interaction framework of the in-house DNS code. This new immersed boundary formulation combines the governing equations from fluid and solid domain and the interface conditions analytically, resulting in a set of meta-equations valid on the full domain. The accuracy of the method in the overlapping region between both domains is enhanced to second order by a correction term taking into account the normal derivative to the surface, as proposed in Maertens and Weymouth. ${ }^{15}$ The resulting equations from the BDIM are applied after the numerical time integration and no modification of the individual solver algorithms are needed.

\section{Computational Setup}

The computational domain is composed by an Eulerian grid for the fluid of dimensions $L_{X}$ by $L_{Y}$, with a refined central area where the membrane is located, as shown in Fig.1. The lower and left boundaries are treated as inlets and an integral characteristic boundary condition for a uniform non-dimensional velocity was applied. At the free-stream upper bound non-reflecting characteristic boundary condition were used and at the right outlet edge the zonal characteristic boundary condition is applied within a finite region of 20 points. Grid resolution and domain size are established by an iterative approach discussed in next section.

The membrane configuration simulated here is based on an experimentally tested model from Rojratsirikul, ${ }^{16}$ composed of a latex sheet mounted onto two rigid aerodynamically-shaped rims at leading and trailing edge. The dimension used for non-dimensionalization of the problem is the actual length of the latex sheet, with the total chord-length of the aerofoil, including mounts, being 1.086 non-dimensional units. The fine region length $L_{M}$ is adjusted to this non-dimensional chord length and the height is $H_{M}=0.1$, covering the maximum height expected for the deflected membrane. In this finer area the points are distributed equidistantly in the wall normal direction with resolution $\Delta y=2.5 \times 10^{-4}$, estimated in order to include at least six Eulerian grid points over the membrane thickness for accuracy of the interpolation between grids. In the streamwise direction the grid is stretched towards the midpoint of the membrane, with a minimum spacing around leading and trailing edge of $\Delta x=5 \times 10^{-4}$ that assures a minimum ratio of 1 : 4 Langrangian to Eulerian points necessary for interpolation. The distribution of points away from this region is stretched toward the boundaries of the domain by means of a 5 th order polynomial function.

The one-dimensional Lagrangian grid for the structural solver includes 100 points along the membrane length. It has been checked with additional simulations that increasing the number of points in this grid does not improve the accuracy of the solution, but increases the computational cost. The membrane length is one non-dimensional unit and the thickness is chosen $h=1.33 \times 10^{-3}$, in order to keep the same thickness/length ratio as the reference case, ${ }^{2}$ and it is represented by the boundary data immersion method mentioned before.

\begin{tabular}{cccccccccc}
\hline \hline Grid & G1 & G2 & G3 & G4 & G5 & G6 & G7 & G8 & G9 \\
\hline$L_{X}$ & 21 & 21 & 21 & 21 & 21 & 31 & 31 & 61 & 61 \\
$L_{Y}$ & 20.1 & 20.1 & 20.1 & 20.1 & 20.1 & 30.1 & 30.1 & 60.1 & 60.1 \\
$N_{X}$ & 597 & 747 & 797 & 897 & 747 & 769 & 957 & 997 & 1097 \\
$N_{Y}$ & 475 & 475 & 475 & 475 & 624 & 487 & 495 & 575 & 775 \\
$N_{X M}$ & 400 & 550 & 600 & 700 & 550 & 550 & 600 & 600 & 600 \\
$N_{Y M}$ & 377 & 377 & 377 & 377 & 526 & 377 & 377 & 377 & 377 \\
\hline
\end{tabular}

Table 1: Parameters for the Eulerian grid refinement and domain size investigation. 


\section{2D direct numerical simulations}

Direct numerical simulations were carried out at $R e=2500$ and $R e=10,000$ for two different angles of attack, 4 and 8 degrees, for uniform velocity inlet condition. The Mach number was set to 0.2 , assuming that the flow behaves incompressibly in this range. The non-dimensional properties of the membrane are the same in all cases: $\rho_{s} h=0.589, E h=50, \delta_{0}=0$ and $C_{d} h=0$. They were chosen from one of the cases investigated by Gordnier ${ }^{2}$ in order to produce comparable results to validate the present computational framework.

\section{A. Steady case: angle of attack 4 degrees, $R e=2500$}

In the first DNS the angle of attack is set to 4 degrees. The membrane is initially flat and is expected to deflect until it achieves a steady state after the initial transient. This case is used to validate the new fluid structure interaction framework of the code and study the influence of the grid resolution in the present problem. To this end six different fluid grids were tested with their details given in Table.1. For grid G1 to G4, the number of point in the streamwise direction $N_{X}$ was increased, starting from G1 with a resolution around the membrane twice that of the reference calculation. ${ }^{2}$ This parameter directly affects the accuracy of the results for the pressure distribution. Increasing the resolution improves the pressure captured over the membrane surfaces and makes the $C_{p}$ distribution smoother. Comparison of mean deflection and mean $C_{p}$ along the chord length in Fig.2 shows that no significant changes in the results are observed when the number of points along the aerofoil length $N_{X M}$ is larger than 600. Since the results from grid G2 already collapse with those from G3 and G4, the number of points $N_{X}$ from this grid is used in grid G5 and the number of points $N_{Y}$ is increased to study the effect of refinement of the wall-normal direction. As can be seen in Fig.2, the wall-normal resolution is sufficient in grid G2. Finally, the influence of the domain size is also investigated comparing results from grids G2 and G6, where the resolution of the central fine region was kept as in G2, and additional points were added toward the far field boundaries in grid G6. Figure 2 presents imperceptible differences in the results, therefore it can be concluded that the distance between the aerofoil and the boundaries established in grid G2 is appropriated for this problem.

Grid G2 seems to capture fluid and structural behaviour with no evidence of under-resolution. The DNS results for this case presents very good agreement with the reference. ${ }^{2}$ The difference in deflection values at the maximum of the curve is just approximately $0.9 \%$, due to a small difference of pressure distribution of approximately $5.2 \%$. In light of these results, this can be considered as a successful validation of the code for steady cases.
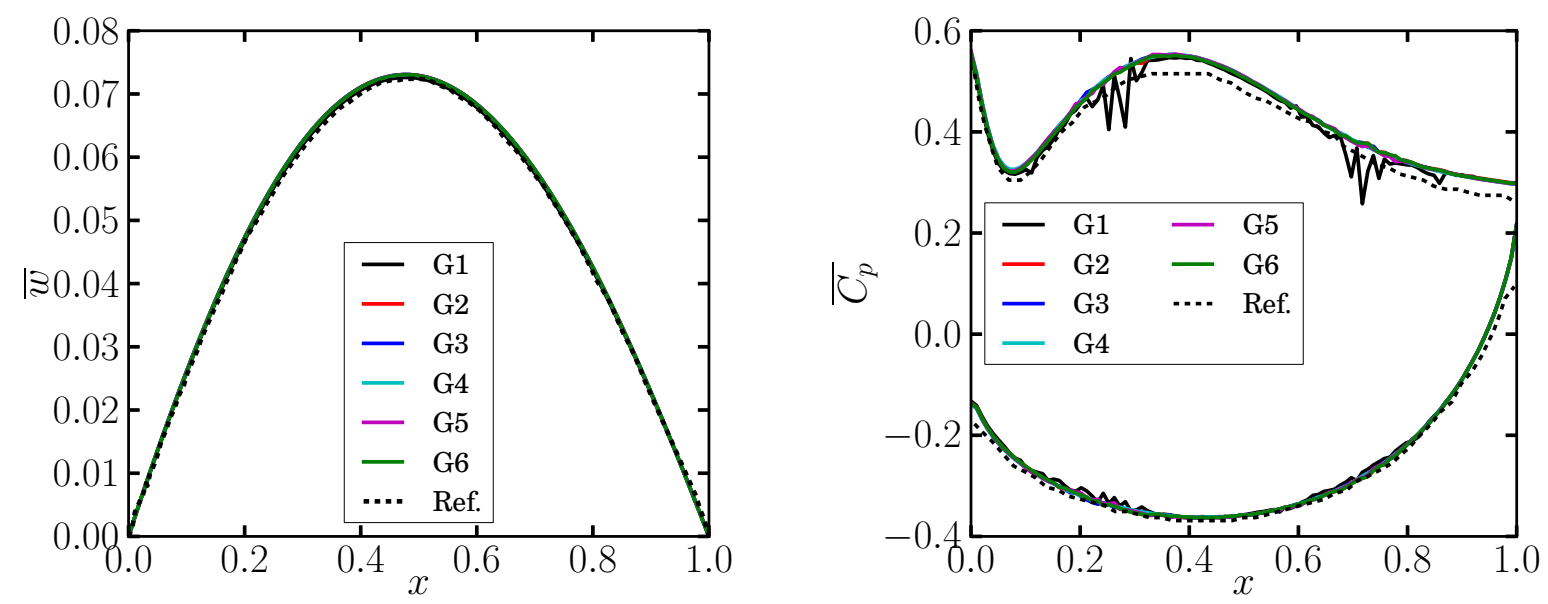

Figure 2: Comparisons of reference case ${ }^{2}$ and DNS results at $R e=2500$ and angle of attack of 4 degrees for different grid resolutions and grid sizes. 


\section{B. Unsteady case: angle of attack 8 degrees, $R e=2500$}

A second set of DNS were performed with 8 degrees angle of attack at the inlet boundary condition. For this case a non-steady but periodic behaviour of the membrane wing is expected. The first of these simulation was performed using grid G3 described previously, assuming that the slightly higher resolution of this grid with respect to G2 would be more suited for unsteady movement of flow and structure. The results obtained were very similar to the corresponding case in the reference ${ }^{2}$ in mean deflection values and $C_{p}$ distributions (Fig.3). The spectra of the perturbations of $w$ and $C_{p}$ at three different locations along the chord in grid G3 were computed from a time series after the transient showing some differences to the reference. The structural and pressure response (Fig.4) contain a dominant frequency for the periodic movement of $f \approx$ 1.41 , very close to the reference case, but two more peaks appear at frequencies $f \approx 0.21$ and $f \approx 1.56$ that are not present in the reference. These secondary frequencies correspond to an observed modulation in the time history for displacement $w$ and pressure value on the upper surface of the membrane. Also, unexpected values of root mean square of pressure were found in the flow field near the domain boundaries. Therefore a new grid, G7, was produced, increasing the length of the domain ten chords in the $x$ and $y$ directions, keeping the interior resolution of grid G3 and adding extra points towards the boundaries. Mean values remain almost exactly the same than in case of grid G3. However the spectral analysis in Fig.5 shows that the perturbations in pressure on the membrane surface and therefore the perturbations in membrane displacement becomes periodic with a single dominant frequency of $f \approx 1.4$. These periodic oscillations seem to be very similar to the third mode standing response found by Gordnier. ${ }^{2}$

In order to ensure the grid-independence of these results two additional grids, G8 and G9, were generated and tested. Grid G8 maintains the same resolution than grid G7, but increasing the domain size to 30 length units in the stream-wise and wall-normal directions, adding additional grid-points. Grid G9 was generated increasing resolution respect to G8, but keeping the same domain size. Spectral analysis of these two cases presented in Fig. 5 demonstrates that the periodic behaviours in deflection and pressure perturbations remain a single dominant frequency of $f \approx 1.4$. Comparison of the mean deflection and mean $C_{p}$ distribution for the four grids used in this section are plotted in Fig.3 showing minimal differences. Geometric details of all the grids are specified in Table 1.

Therefore, the conclusion of this study is that results are grid-independent and that resolution and dimensions of grid G7 are appropriate for investigation of the unsteady configuration under consideration, showing good agreement with the reference case. The difference in deflection values at the maximum of the curve is just approximately $1.5 \%$, caused by difference of pressure distribution values of approximately $2.7 \%$, as can be seen in Fig.3.
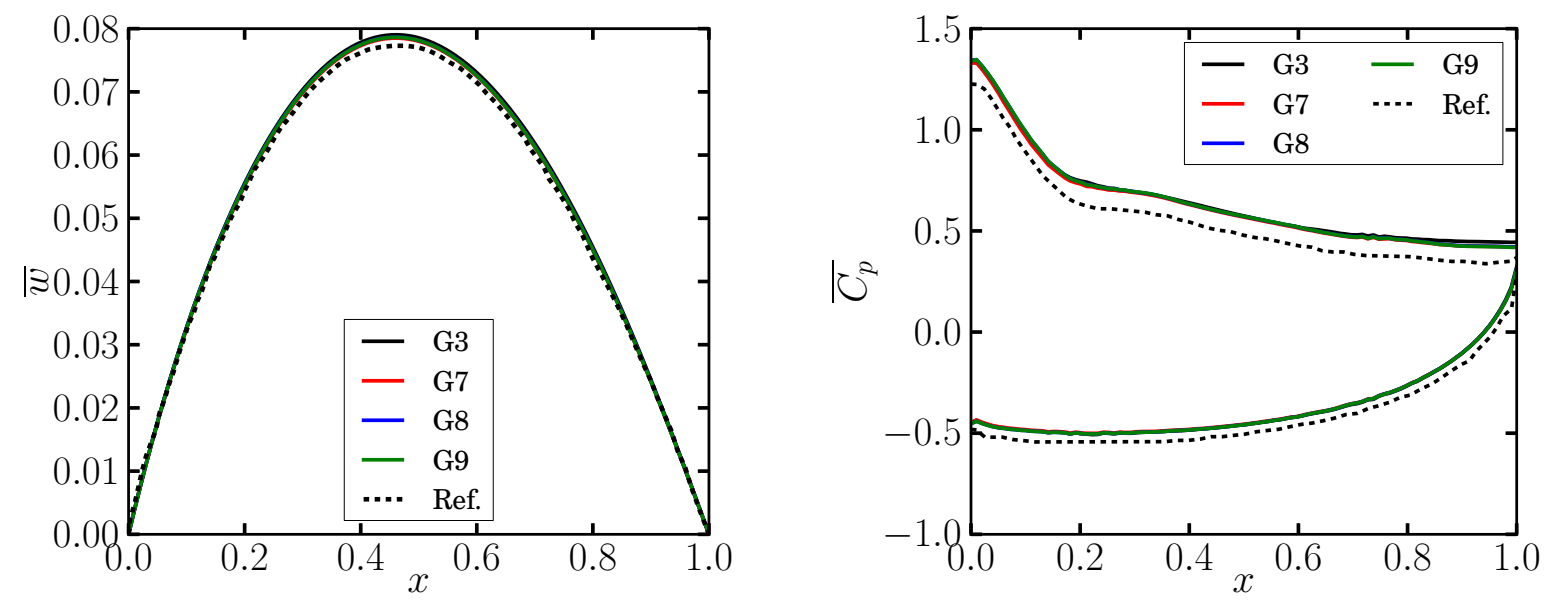

Figure 3: Comparisons of mean deflection and surface pressure coefficient from DNS results in different grids and reference, ${ }^{2}$ at $R e=2500$ and angle of attack of 8 degrees. 

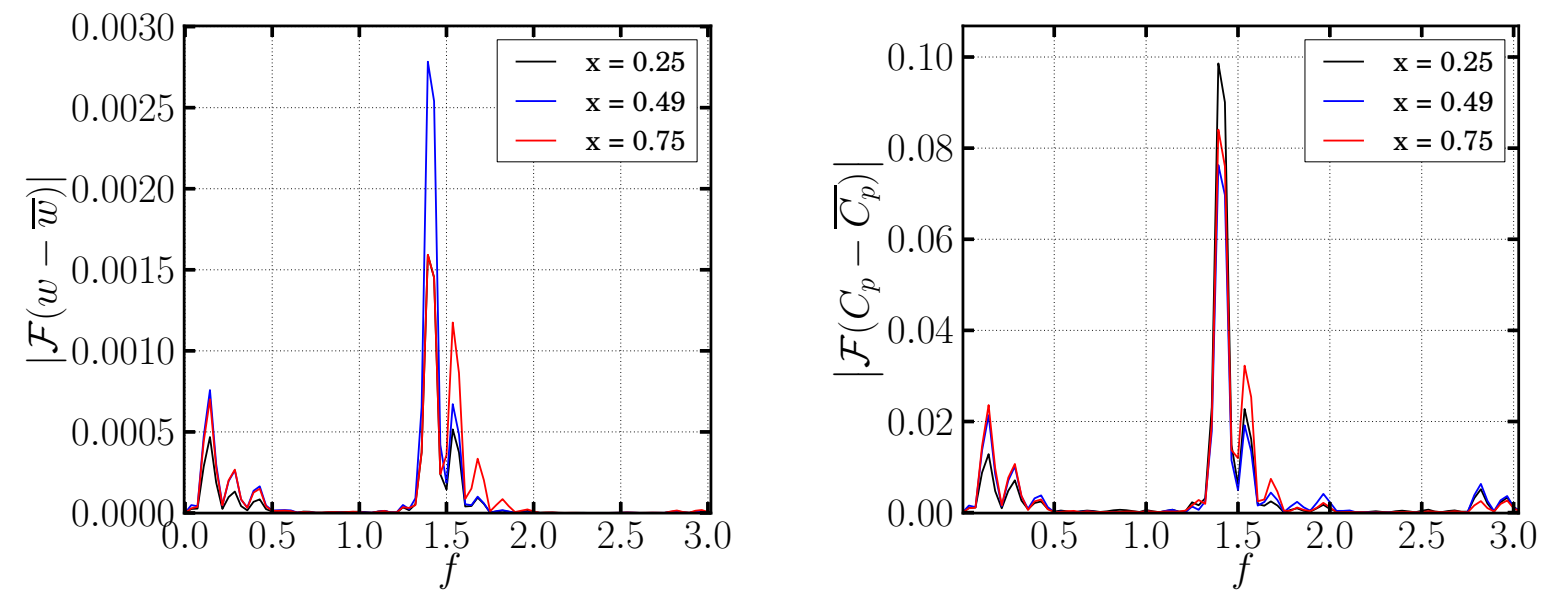

Figure 4: Spectral analysis from DNS results at $R e=2500$ and angle of attack of 8 degrees in grid G3.
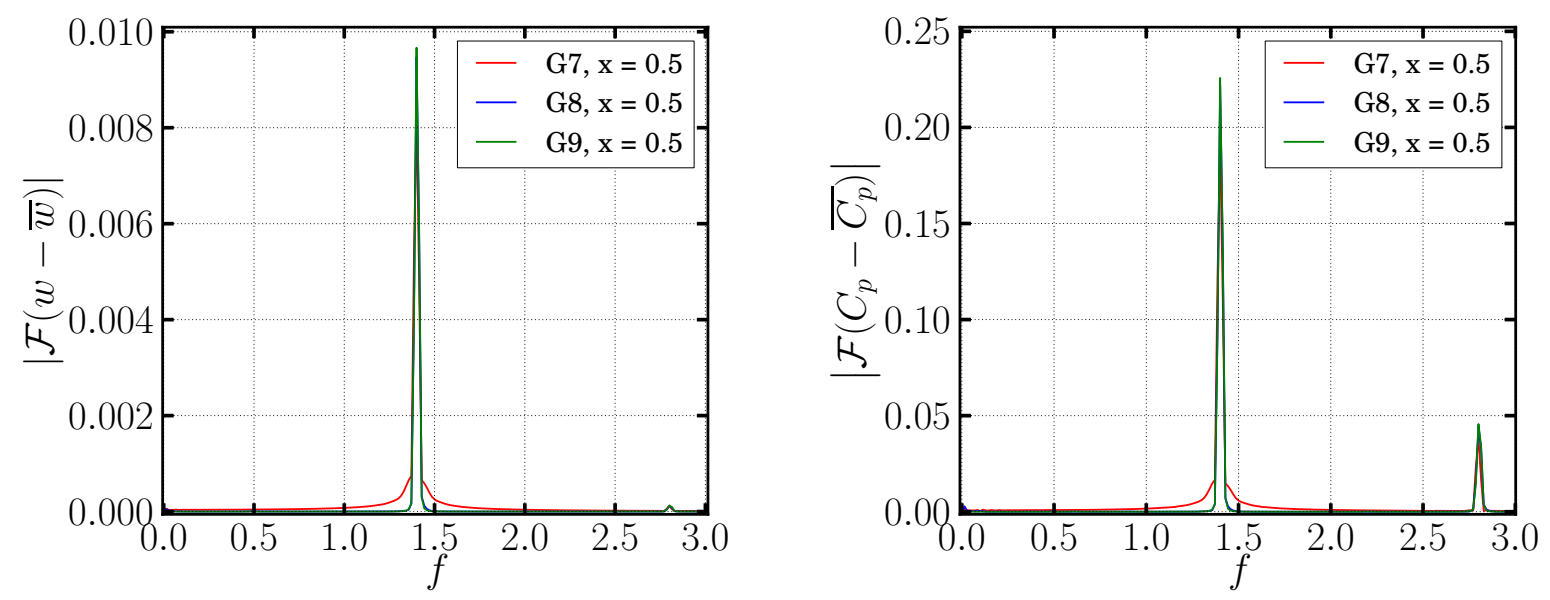

Figure 5: Comparisons of spectral analysis from DNS results at $R e=2500$ and angle of attack of 8 degrees in grids G7, G8 and G9.

\section{Unsteady case: angle of attack 8 degrees, $R e=10,000$}

Another two-dimensional case was studied maintaining the angle of attack at 8 degrees and increasing Reynolds number up to 10,000 , closer to a more realistic flight situation for MAVs. Under these conditions the flow is expected to become more complex and unstable than in the previous cases, and it is suggested in references ${ }^{2}$ that it could be the limit for the two-dimensional laminar flow assumption. Two DNS simulations were carried out using grid G7 and G9 with characteristics specified in Table 1, varying resolution of the computational domain outside of the refined region. The resolution in the proximity of the membrane was stabilised for the lower Reynolds number cases attending the requirements of interpolation between Lagrangian and Eulerian grids and accuracy when resolving fluid structures. The grid spacing determined for those cases is still on the same range of values of those used in references studies ${ }^{17},{ }^{18}$ for aerofoils at $R e=10,000$ and $R e=50,000$, therefore the resolution of the refined region for G7 and G9 is considered 

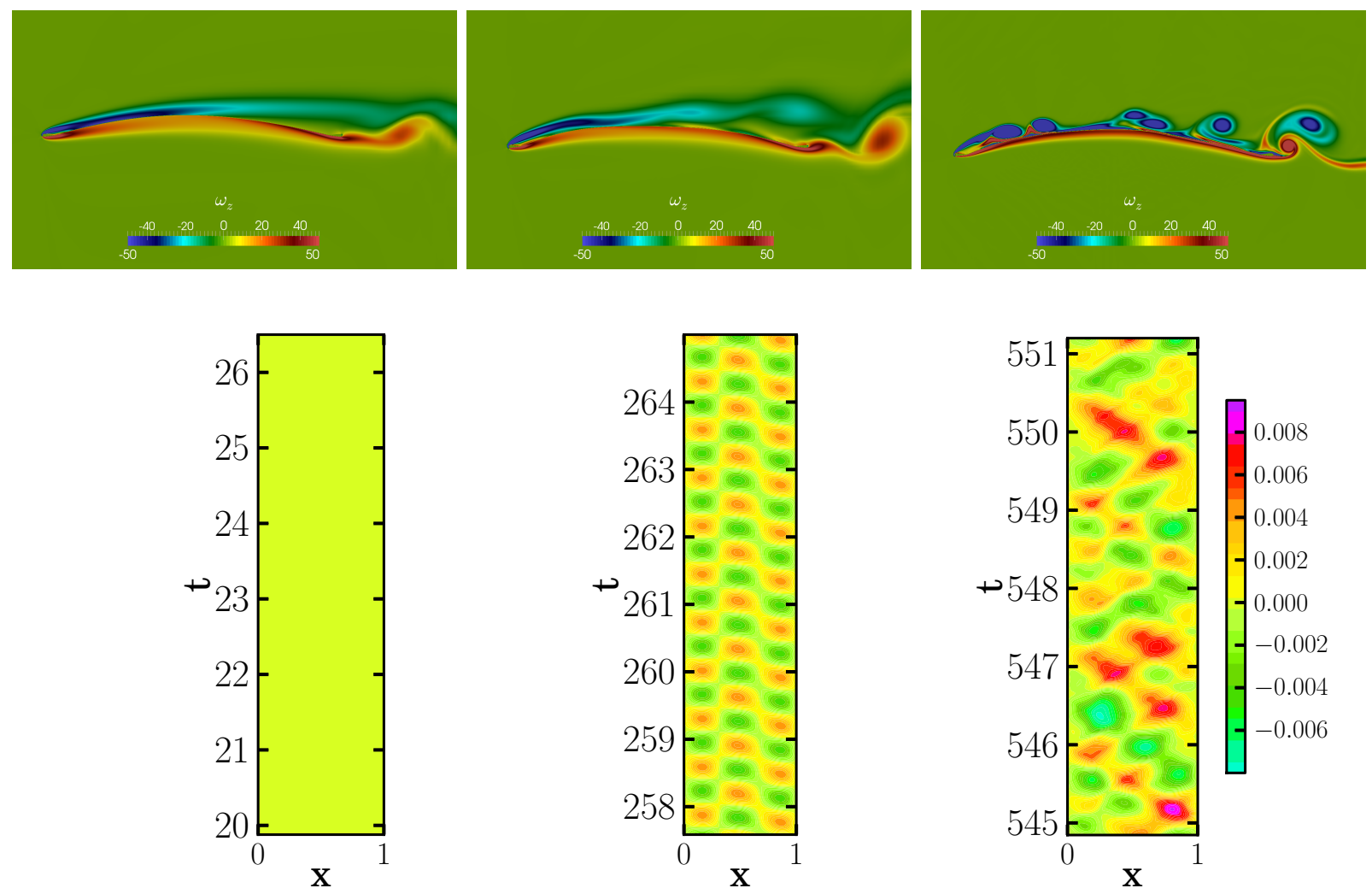

Figure 6: Vorticity and time-history diagrams along the chord length from DNS results at: $R e=2500$ and angle of attack of 4 degrees (left), $R e=2500$ and angle of attack of 8 degrees (centre) and $R e=10,000$ and angle of attack of 8 degrees (right).

sufficiently good for the case under study here, as it will be shown later.

Results for mean deflection of the membrane and $C_{p}$ distribution for DNS in both grids are shown in Fig.7 demonstrating good agreement with the reference also in this case. The difference of pressure distribution values at the maximum deflection location is approximately $1.8 \%$, causing a difference in $w$ of just approximately $0.9 \%$. The spectral analysis of the time signal for $w$ and $C_{p}$ at the midpoint of the membrane together with the $C_{p}$ time signal of a point in the near wake in Fig.7 reflects the change of the dynamics of flow and membrane. In this case, in order to represent three time series in the same plot, the values of the Fourier series coefficients were non-dimensionalized whit the root mean squared of their corresponding time signal. As expected, the purely periodic behaviour of oscillations disappears and their energy is distributed in a wider range of frequencies. The most energetic frequencies for the oscillations of the membrane are confined to the lower part of the spectra at $f \approx 0.58,0.8,1.2,1.6,2.1$ and 2.7, the first three of them coinciding with the linear model frequencies mentioned by Gordnier. ${ }^{2}$ The dominant frequencies for pressure oscillations in this range correlate with those from the membrane, showing peaks at $f \approx 0.6,0.9,1.2,1.6,2.1$ and 2.7. However, the range of frequencies containing a significant amount of energy for the pressure fluctuations extends up to $f \approx 8$ in a similar trend to the one observed for the spectra at the wake. This shows the intimate correlation between flow and membrane movement, even for more complex dynamics caused by higher Reynolds number, and it also indicates the influence of further vortex shedding at the trailing edge and near wake on pressure fluctuations on the surface of the membrane. This spectral analysis also proves the validity of the initial assumption that the time lag between $w$ and $p$ inherent on the current formulation is negligible. Results from this analysis are very similar for both tested grids, concluding that G7 is appropriate for two-dimensional simulations at this Reynolds number.

To finalize this section, vorticity contours of all the two-dimensional cases, together with time-history diagrams along the chord length are plotted in Fig.6 showing changes in dynamics of the fluid-structural coupled problem with angle of attack and Reynolds number, as well as qualitative agreement of these 
quantities with the reference cases.

These 2D simulations are suitable to validate the new computational framework, perform grid resolution study and extract a first characterization of the flow-structural behaviour. When Reynolds number is increased vortices convecting over the surface of the membrane appear clearly defined and a wide range of significant temporal scales are observed in the spectral analysis. Therefore the next natural step is to carry out further investigations on the flow motion development when the artificial restrictions imposed by two-dimensionality are removed. Furthermore, the availability of the $2 \mathrm{D}$ flow fields now allows to investigate the instability behaviour.
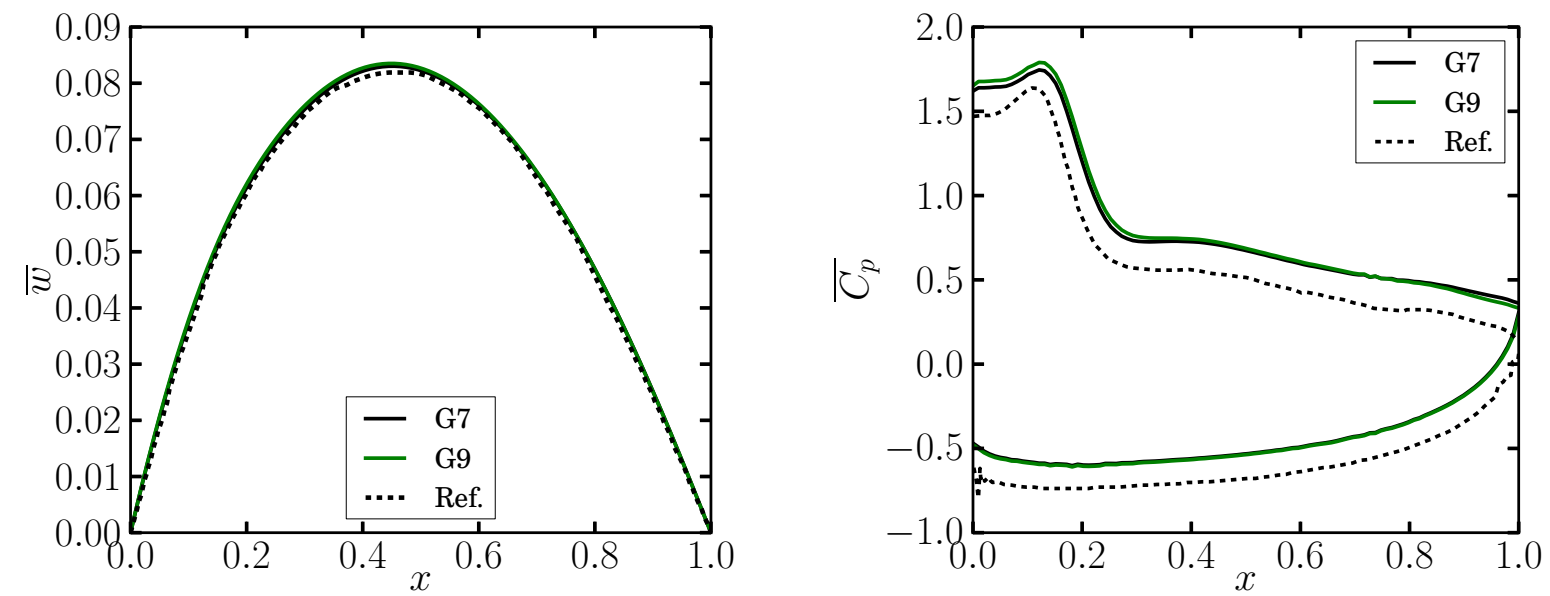

Figure 7: Comparisons of mean deflection and surface pressure coefficient from DNS results in different grids and reference, ${ }^{2}$ at $R e=10,000$ and angle of attack of 8 degrees.
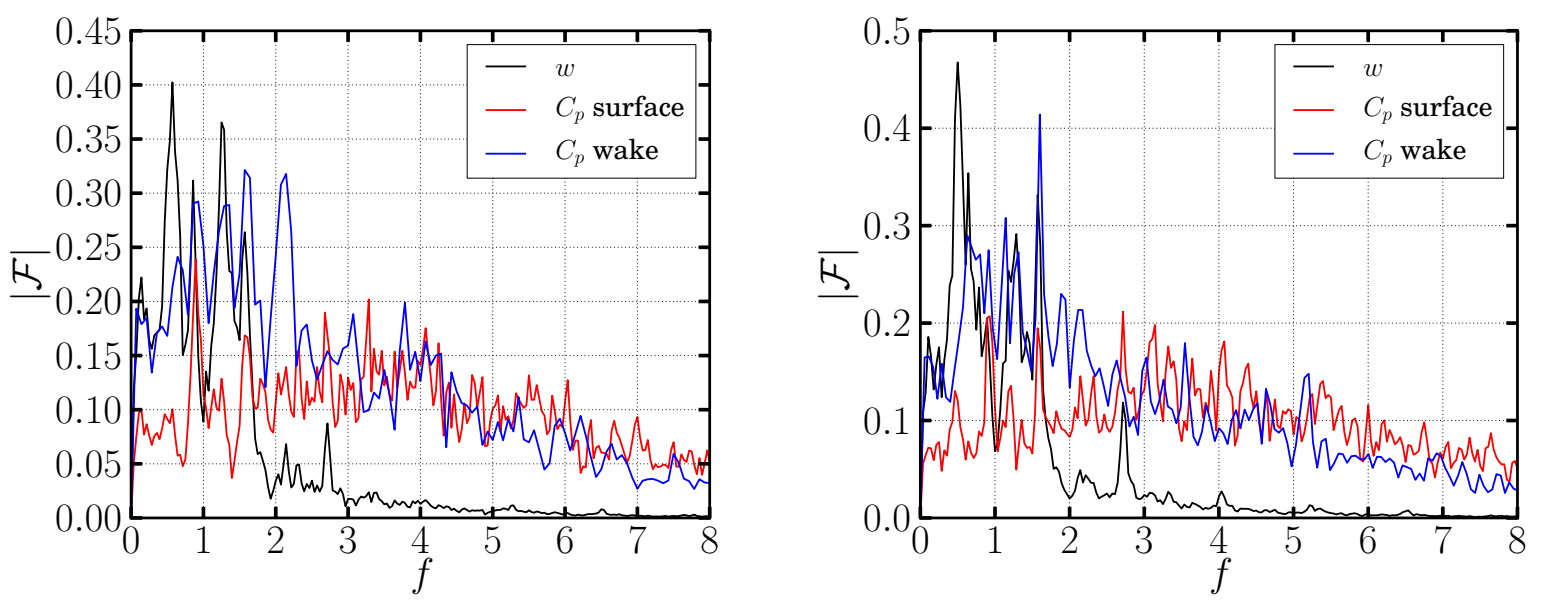

Figure 8: Spectral analysis from DNS results at $R e=10,000$ and angle of attack of 8 degrees at the midpoint of the membrane $(x=0.5)$ in grid G7 (left) and G9 (right). 


\section{3D stability analysis}

The flow field and membrane state from the $2 \mathrm{D}$ cases at angle of attack 8 degrees were taken as initial condition for three dimensional simulations at $R e=2500$ and $R e=10,000$. The two dimensional flow solution was initially extended in the z-direction and initial perturbations of the spanwise velocity component $\left(u_{3}\right)$ were introduced in the full fluid domain in the form of white noise with a maximum amplitude of $1 \times 10^{-8}$ in order to identify regions of instability. The fluid equations are discretized using Fourier transforms in the homogeneous and periodic spanwise direction. Eight Fourier modes were used, which most likely is not sufficient for a well resolved 3D DNS but is appropriate for the stability investigation. Two different spanwise domain widths, $L_{Z}=0.2$ and $L_{Z}=0.5$, were used in the simulations at both Reynolds numbers based on reference studies for aerofoils ${ }^{18}$ and grid G9 was chosen due to its higher resolution, more suitable for possible emerging complex fluid structures. The initial $u_{3}$-perturbations are superimposed on the initial flow field for all Fourier modes $k \geqslant 1$. On the structural side, spanwise variations of the membrane are not permitted and the structural equation solved is still the one presented in section II.B. The pressure term $\Delta p$ in these $3 \mathrm{D}$ cases corresponds to the pressure difference between upper and lower surfaces of the membrane, averaged in the spanwise direction at every time step. The properties of the membrane are the same than in the previous cases: $\rho_{s} h=0.589, E h=50, \delta_{0}=0$ and $C_{d} h=0$. The two-dimensional geometry of the deflected membrane is extruded in the spanwise direction and just the flow perturbations are allowed to develop in $3 \mathrm{D}$.

\section{A. Case 1: angle of attack 8 degrees, $R e=2500$}

The first 3D simulation was initialized with the 2D solution from the case presented in section IV.B and $u_{3^{-}}$ perturbations were superimposed on the initial condition. After the first time-step no additional disturbances are added and the simulation is advanced in time while observing the evolution of small initial perturbations in the proximity of the membrane. In the absence of instability mechanisms the perturbation will decay in amplitude and will be convected downstream, whereas if the disturbances amplitude increases, with or without being convected, it will be an indication of instability regions and the characteristics of this process can be further analysed.

The evolution of the initial perturbations is observed in the temporal series of the amplitude of the Fourier modes $|\mathcal{F}|$ at different locations close to the membrane surface. For this low Reynolds number case it was observed that the initial disturbances are quickly damped and convected downstream in the full domain, resulting in low values in $u_{3}$ only in the region of the wake. Time signals of the amplitude of spanwise velocity perturbations Fourier modes $\left|\mathcal{F}\left(u_{3}\right)\right|$ were recorded at two locations close to the surface of the membrane, at $(x, y)=(-0.7,0.0)$ in the vicinity of the recirculation bubble close to the leading edge, and at $(x, y)=(-0.3,0.0)$ in the separated region. In Fig.9 and Fig.10 it can be observed that all higher modes for spanwise velocity perturbations show temporal decay indicating absence of instability. Therefore the flow stays essentially two dimensional, implying that the $2 \mathrm{D}$ simulations results are representatives. The results for the two spanwise domain sizes investigated are very similar, indicating no influence of length in the spanwise dimension.

\section{B. Case 2: angle of attack 8 degrees, $R e=10,000$}

A second set of three dimensional simulations was carried out at $R e=10,000$ initialized with the solution from the $2 \mathrm{D}$ case of section IV.C upon which initial $u_{3}$ perturbations were superimposed in the same manner as in the case above.

For the first simulation the spanwise width of the computational domain was $L_{Z}=0.2$. In this case initial perturbations in the free stream flow were also quickly damped and convected downstream, however in the proximity of the membrane surface and particularly in the recirculation bubble close to the leading edge the amplitude of the perturbation remains initially constant for less than one time unit and then starts increasing. This suggests that the recirculation region becomes an amplifier for the perturbations. According to the spectra in Fig. 8 the dominant frequency of pressure fluctuations is $f \approx 1.6$. This frequency determines a periodic vortex shedding cycle of a pair of vortices that emerge from the proximity of the leading edge, 

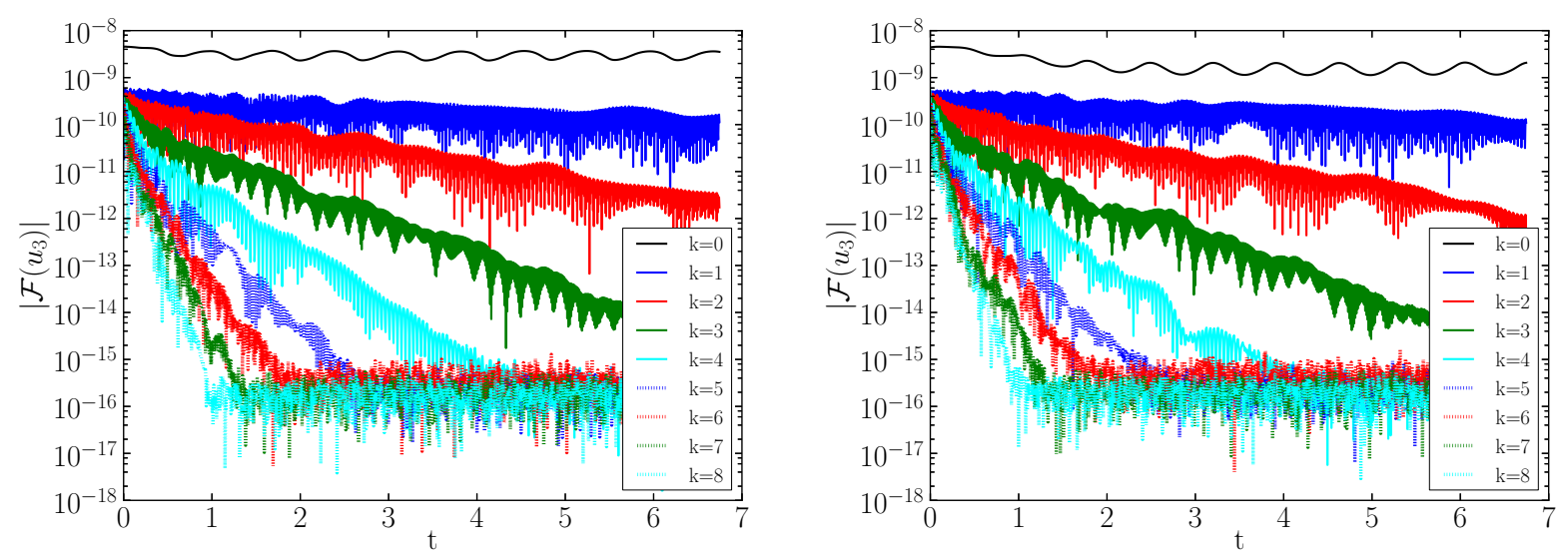

Figure 9: Temporal evolution of spanwise Fourier modes of the z-component of velocity at $(x, y)=(-0.7,0.0)$ (left) and $(x, y)=(-0.3,0.0)$ (right) at $\mathrm{Re}=2500$ for spanwise domain width $L_{Z}=0.2$.
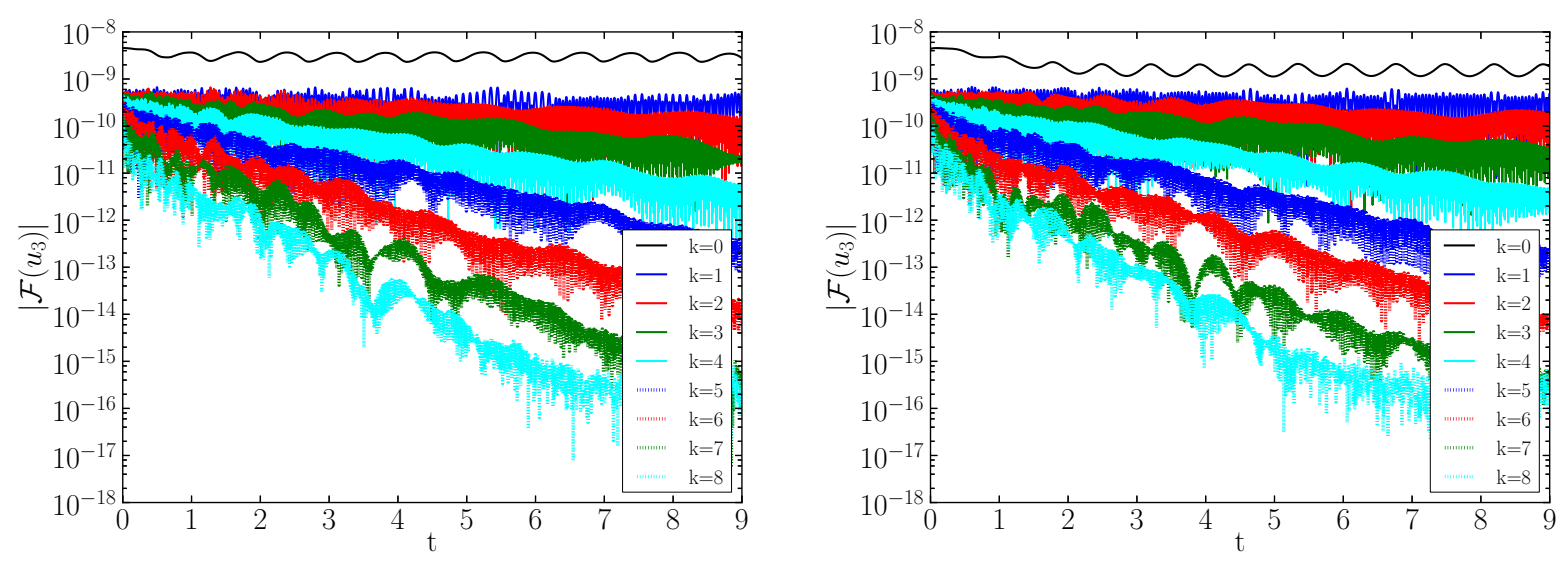

Figure 10: Temporal evolution of spanwise Fourier modes of the z-component of velocity at $(x, y)=$ $(-0.7,0.0)$ (left) and $(x, y)=(-0.3,0.0)$ (right) at $\mathrm{Re}=2500$ for spanwise domain width $L_{Z}=0.5$.

detach from this recirculation region and are convected over the membrane surface, until the next pair of vortices is generated. One of the vortices of this pair always stays very close to the membrane surface while being convected whereas the other one is slightly lifted above the membrane while moving downstream. Iso-contours of $\left|u_{3}\right|$ at the midspan plane are plotted along one vortex shedding cycle in Fig. 11 illustrating the perturbation amplification process: fluid particles initially recirculating inside of the leading edge vortex have low values of spanwise velocity that increases during the time that the vortex stays attached to the leading edge. While the vortex detaches from the leading edge and is convected downstream the values of $\left|u_{3}\right|$ continue increasing in its core as well as in its surrounding shear layer. For the lower vortex of the pair the strongest growth of $\left|u_{3}\right|$ appears in the shear layer between the vortex and the membrane surface. However, for the upper vortex the strongest growth appears far from the membrane surface, on its surrounding shear layer between vortex and freestream. At the same time that the vortices convect downstream instabilities in both shear layers mix with the ones in the core while increasing in amplitude.

Figure 12 shows the evolution of the initial perturbations at $(x, y)=(-0.7,0.0)$, inside of the amplification region, and $(x, y)=(-0.3,0.0)$, downstream of the separation point. It can be observed that initially only the lower Fourier modes $\mathrm{k}=1,2,3$ and 4 grow, with mode $\mathrm{k}=2$ being the most amplified. The higher modes initially decay and only increase at a later stage, when they start interacting with the lower modes and the amplitude of perturbations is several order of magnitude higher than the initial perturbation. At 

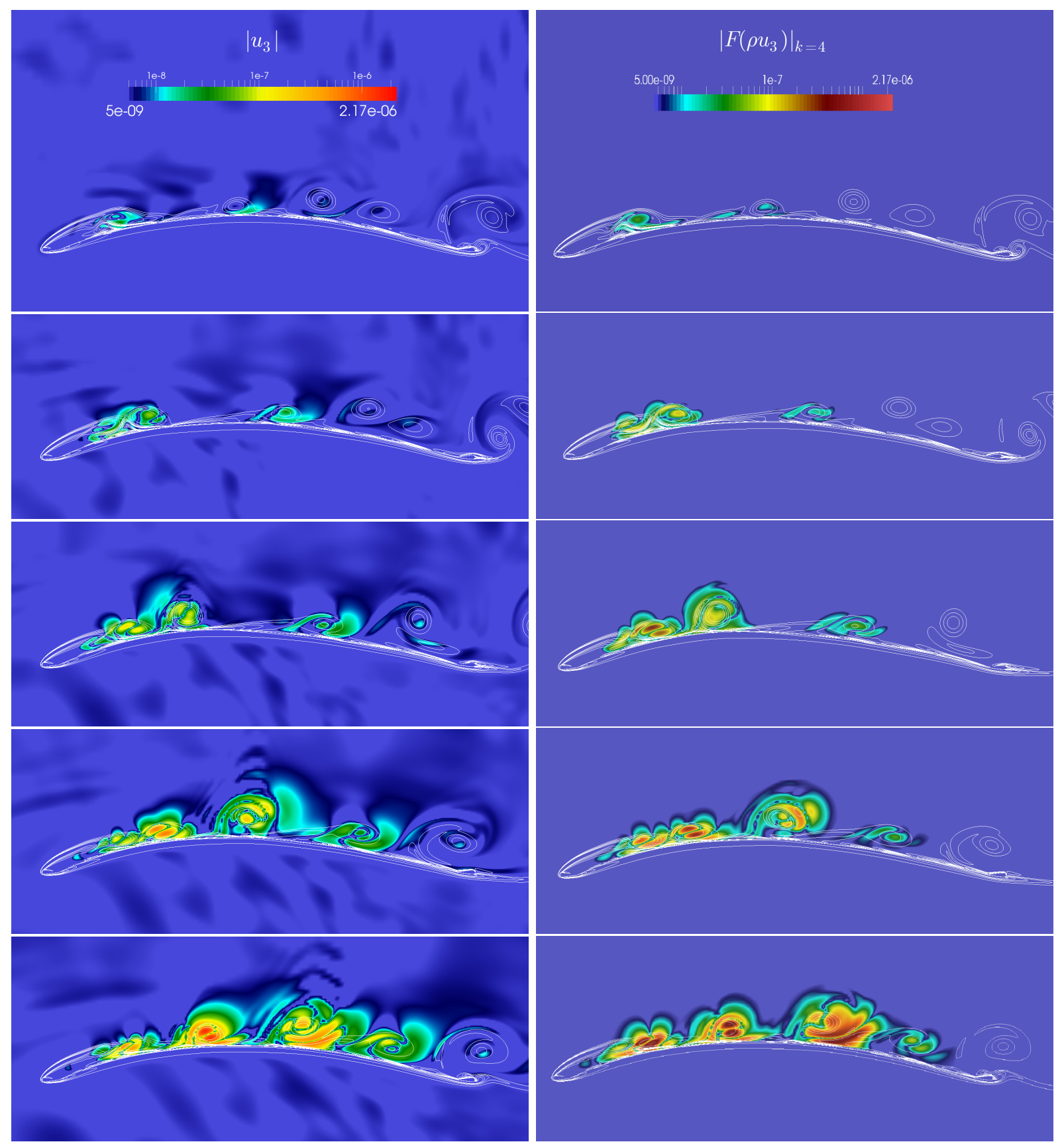

Figure 11: Left: Iso-contours of spanwise velocity perturbation amplitude with lines of constant $\omega_{z}$ at $\mathrm{Re}=10,000$ for spanwise domain width $L_{Z}=0.2$. Right: Iso-contours of the amplitude of the fourth Fourier mode of $\left|u_{3}\right|$ with lines of constant $\omega_{z}$ at $\operatorname{Re}=10,000$ for spanwise domain width $L_{Z}=0.5$. Five phases of the vortex shedding cycle are shown in the two cases, from top to bottom: $\tau=0, \tau=0.25 T, \tau=0.5 T$, $\tau=0.75 T, \tau=T$.

that stage, the simulation was stopped as the interest of this study is only the stability behaviour of the flow and it is not desired to conduct a DNS with insufficient resolution in the spanwise direction. The decay trend observed in the higher Fourier mode indicates that for $L_{Z}=0.2$ the chosen number of modes is sufficiently large to detect the most unstable ones, since perturbations with smaller scale represented by modes higher than $\mathrm{k}=8$ would not increase in amplitude. However, the fact that the most amplified modes are $\mathrm{k}=1$ and 2 could be an indication of insufficient spanwise width for the computational domain. Therefore a second simulation was performed with $L_{Z}=0.5$. 

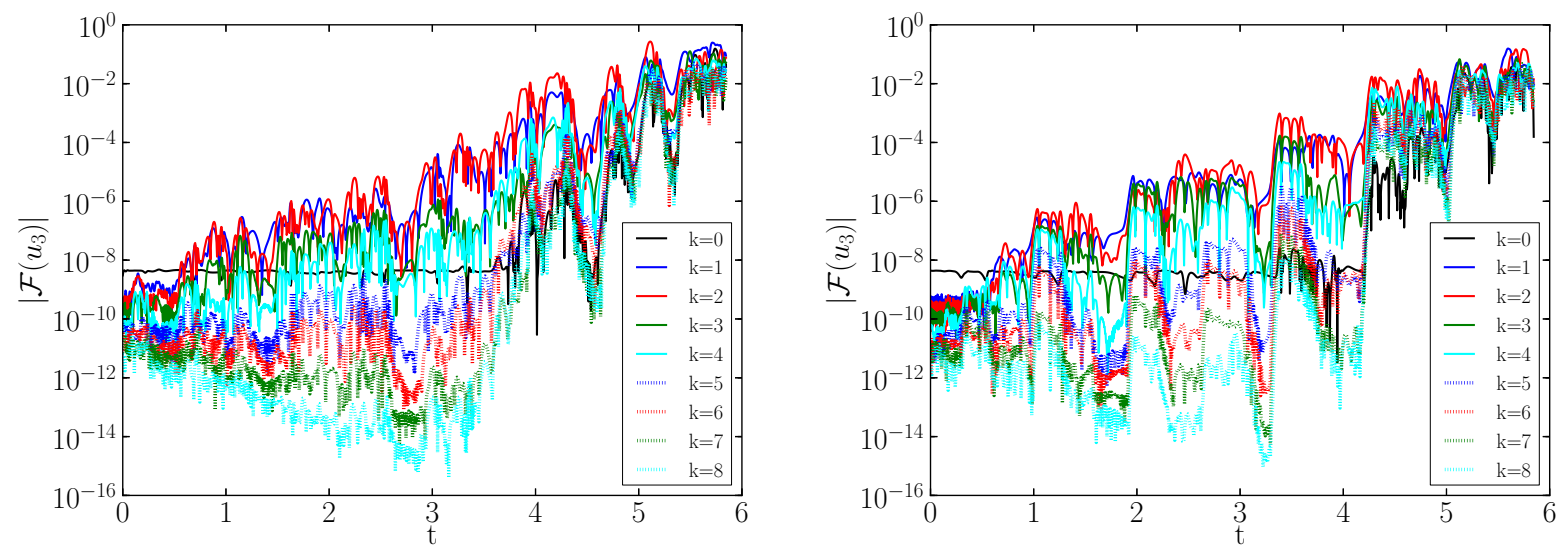

Figure 12: Temporal evolution of spanwise Fourier modes of z-component velocity at $(x, y)=(-0.7,0.0)$ (left) and $(x, y)=(-0.3,0.0)$ (right) at $\operatorname{Re}=10,000$ for spanwise domain width $L_{Z}=0.2$.

In this second simulation with wider computational domain the mechanisms for the perturbations growing along vortex shedding cycle remains qualitatively identical to the one described before, however differences were found in the temporal growth of the Fourier modes. As it is observed in Fig. 13 in this case modes $\mathrm{k}=4,3$ and 5 exhibit the largest growth rate, in that order. Mode $\mathrm{k}=5$ for this spanwise length is the corresponding mode to $\mathrm{k}=2$ of the previous simulation but, although it is one of the most amplified in this case, it is not the dominant one. Mode $\mathrm{k}=1$ is the least amplified in this case but now none of the modes decay after the initial state, due to the coarser resolution in Fourier space for this case. It can be concluded from the study presented here that the most unstable Fourier mode for this case is mode $\mathrm{k}=4$ for a spanwise width of $L_{Z}=0.5$, which corresponds to perturbations with wavelength=0.125. In Fig.11 iso-contours show the shape of this Fourier mode along the same cycle phases presented for the perturbation amplitude. Comparison with the results from the case of $L_{Z}=0.2$ indicate that the different computational domain length in the spanwise direction does not have an effect on the mechanism of amplification of perturbations but for quantitatively accurate results this dimension needs to be larger than $L_{Z}=0.2$ in order to capture the longest wavelength structures.
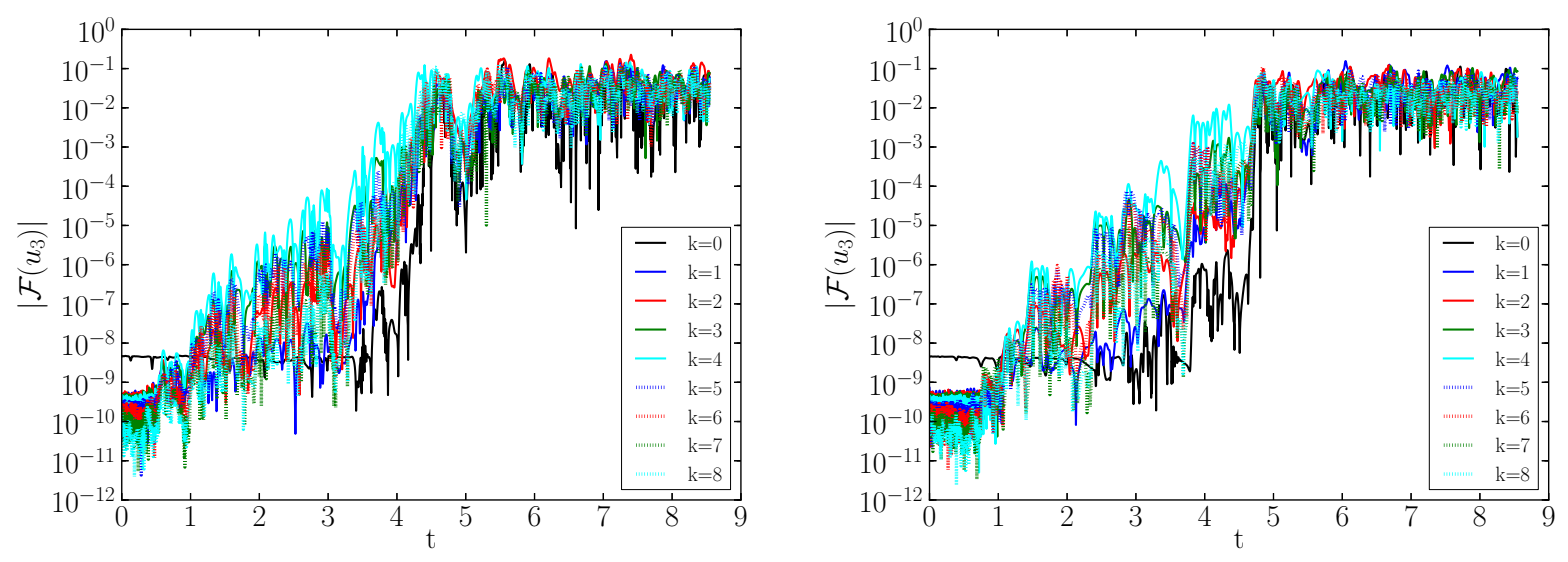

Figure 13: Temporal evolution of spanwise Fourier modes of z-component velocity at $(x, y)=(-0.7,0.0)$ (left) and $(x, y)=(-0.3,0.0)$ (right) at $\mathrm{Re}=10,000$ for spanwise domain width $L_{Z}=0.5$. 


\section{Conclusion}

Direct numerical simulation results for the fluid-structure interaction problem of a membrane wing represented via a new boundary data immersion technique were presented for a range of two dimensional cases. The cases for the flow parameter $R e=2500$ and angle of attack 4 and 8 degrees show respectively steady and periodical behaviours for fluctuations of the membrane deflection and flow pressure distribution around its surface that agree with previous studies. ${ }^{2}$ Results analysed in these cases confirm the validity and accuracy of the new computational set-up for the problem under study. When the Reynolds number was increased up to $R e=10,000$ for the case with angle of attack of 8 degrees the fluid and membrane motion became more chaotic showing a larger range of dominant frequencies in its spectral analysis. Even in this case of complex dynamics, an intimate relation between structural and flow movement was observed through the correlation in dominant frequencies for the spectra of pressure and membrane deflection oscillations.

The availability of the 2D flow fields from the DNS allowed the investigation of flow stability with respect to initial perturbations. The solutions of the 2D DNS were used as initial conditions for the new 3D simulations upon which initial perturbations in spanwise velocity in the form of white noise were introduced. The first case investigated with $R e=2500$ and angle of attack of 8 degrees showed absence of instabilities. Therefore in this case 2D DNS solutions are representative of the overall flow behaviour. In the second case studied, with $R e=10,000$ and angle of attack of 8 degrees, the recirculation bubble close to the leading edge of the membrane was found to be acting as an amplifier of the perturbations. Analysis of the temporal growth of the perturbations of spanwise Fourier modes reveals that mode $\mathrm{k}=4$ for spanwise width $L_{Z}=0.5$ is the most unstable, corresponding to fluid structures of wavelength 0.125 .

\section{Acknowledgments}

The authors are grateful for the funding provided by EPSRC under grant number EP/J001465/1. Thanks as well to all the family of HiPSTAR/FAT developers at the University of Southampton.

\section{References}

\footnotetext{
${ }^{1}$ Jaworski, J. W. and Gordnier, R. E., "High-order simulations of low Reynolds number membrane airfoils under prescribed motion," Journal of Fluids and Structures, Vol. 31, May 2012, pp. 49-66.

${ }^{2}$ Gordnier, R. E., "High fidelity computational simulation of a membrane wing airfoil," Journal of Fluids and Structures, Vol. 25, No. 5, July 2009, pp. 897-917.

${ }^{3}$ Gordnier, R. E. and Attar, P. J., "Implicit LES simulations of a low Reynolds numeber flexible membarne wing Airfoil," 47th AIAA Aerospace Science Meeting, Vol. 579, 2009.

${ }^{4}$ Ferreira de Sousa, P. J. S. A. and Allen, J. J., "Thrust efficiency of harmonically oscillating flexible flat plates," Journal of Fluid Mechanics, Vol. 674, March 2011, pp. 43-66.

${ }^{5}$ White, F. M., Viscous Fluid Flow, McGraw Hill, 1991.

${ }^{6}$ Carpenter, M. H., Nordström, J., and Gottlieb, D., "A Stable and Conservative Interface Treatment of Arbitrary Spatial Accuracy," Journal of Computational Physics, Vol. 148, No. 2, Jan. 1999, pp. 341-365.

${ }^{7}$ Kennedy, C. A., Carpenter, M. H., and Lewis, R. M., "Low-storage, explicit Runge-Kutta schemes for the compressible Navier-Stokes equations," Applied Numerical Mathematics, Vol. 35, 2000, pp. 177-219.

${ }^{8}$ Kennedy, C. A. and Gruber, A., "Reduced aliasing formulations of the convective terms within the NavierStokes equations for a compressible fluid," Journal of Computational Physics, Vol. 227, No. 3, Jan. 2008, pp. 1676-1700.

${ }^{9}$ Bogey, C., de Cacqueray, N., and Bailly, C., "A shock-capturing methodology based on adaptative spatial filtering for high-order non-linear computations," Journal of Computational Physics, Vol. 228, No. 5, March 2009, pp. $1447-1465$.

${ }^{10}$ Jones, L. E., Numerical studies of the flow around an airfoil at low Reynolds number., Ph.D. thesis, University of Southampton, 2007.

${ }^{11}$ Sandberg, R. D. and Sandham, N. D., "Nonreflecting zonal characteristic boundary condition for direct numerical simulation of aerodynamic sound," AIAA Journal, Vol. 44, No. 2, Feb. 2006, pp. 402-405.

${ }^{12} \mathrm{Kim}$, J. W. and Sandberg, R. D., "Efficient parallel computing with a compact finite difference scheme," Computers 8 Fluids, Vol. 58, April 2012, pp. 70-87.

${ }^{13}$ Weymouth, G. and Yue, D. K., "Boundary data immersion method for Cartesian-grid simulations of fluid-body interaction problems," Journal of Computational Physics, Vol. 230, No. 16, July 2011, pp. 6233-6247.

${ }^{14}$ Schlanderer, S. C. and Sandberg, R. D., "DNS of an Airfoil at Angle of Attack with an Elastic Trailing-Edge Extension," 19th Australasian Fluid Mechanics Conference, Dec. 2014.

${ }^{15}$ Maertens, A. P. and Weymouth, G. D., "Accurate Cartesian-grid simulations of near-body flows at intermediate Reynolds numbers," under review for publication in Journal of Computational Physics, 2013.
} 
${ }^{16}$ Rojratsirikul, P., Wang, Z., and Gursul, I., "Unsteady fluidstructure interactions of membrane airfoils at low Reynolds numbers," Experiments in Fluids, Vol. 46, No. 5, Feb. 2009, pp. 859-872.

${ }^{17}$ Jones, L. E. and Sandberg, R. D., "Numerical analysis of tonal airfoil self-noise and acoustic feedback-loops," Journal of Sound and Vibration, Vol. 330, No. 25, Dec. 2011, pp. 6137-6152.

${ }^{18}$ Jones, L. E., Sandberg, R. D., and Sandham, N. D., "Direct numerical simulations of forced and unforced separation bubbles on an airfoil at incidence," Journal of Fluid Mechanics, Vol. 602, April 2008, pp. 175-207. 